\title{
Reconstruction of Three Dimensional Convex Bodies from the Curvatures of Their Shadows
}

\author{
Rafik Aramyan ${ }^{1,2}$ \\ ${ }^{1}$ Russian-Armenian (Slavonic) University, Yerevan, Armenia \\ ${ }^{2}$ Institute of Mathematics Armenian Academy of Sciences, Yerevan, Armenia \\ Email: rafikaramyan@yahoo.com
}

Received 22 February 2015; accepted 22 May 2015; published 25 May 2015

Copyright (C) 2015 by author and Scientific Research Publishing Inc.

This work is licensed under the Creative Commons Attribution International License (CC BY). http://creativecommons.org/licenses/by/4.0/

(c) (i) Open Access

\begin{abstract}
In this article, we study necessary and sufficient conditions for a function, defined on the space of flags to be the projection curvature radius function for a convex body. This type of inverse problems has been studied by Christoffel, Minkwoski for the case of mean and Gauss curvatures. We suggest an algorithm of reconstruction of a convex body from its projection curvature radius function by finding a representation for the support function of the body. We lead the problem to a system of differential equations of second order on the sphere and solve it applying $a$ consistency method suggested by the author of the article.
\end{abstract}

\section{Keywords}

Integral Geometry, Convex Body, Projection Curvature, Support Function

\section{Introduction}

The problem of reconstruction of a convex body from the mean and Gauss curvatures of the boundary of the body goes back to Christoffel and Minkwoski [1]. Let $F$ be a function defined on 2-dimensional unit sphere $S^{2}$. The following problems have been studied by E. B. Christoffel: what are necessary and sufficient conditions for $F$ to be the mean curvature radius function for a convex body. The corresponding problem for Gauss curvature is considered by H. Minkovski [1]. W. Blaschke [2] provides a formula for reconstruction of a convex body B from the mean curvatures of its boundary. The formula is written in terms of spherical harmonics.

A. D. Aleksandrov and A. V. Pogorelov generalize these problems for a class of symmetric functions $G\left(R_{1}, R_{2}\right)$ of principal radii of curvatures (see [3]-[5]). 
Let $\mathbf{B} \subset \mathbf{R}^{n}$ be a convex body with sufficiently smooth boundary and let $R_{1}(\omega), \cdots, R_{n-1}(\omega)$ signify the principal radii of curvature of the boundary of $\mathbf{B}$ at the point with outer normal direction $\omega \in \mathrm{S}^{n-1}$. In $n$-dimensional case, a Christoffel-Minkovski problem is posed and solved by Firay [6] and Berg [7] (see also [8]): what are necessary and sufficient conditions for a function $F$, defined on $S^{n-1}$ to be function $\sum R_{i_{1}}(\omega) \cdots R_{i_{p}}(\omega)$ for a convex body, where $1 \leq p \leq n-1$ and the sum is extended over all increasing sequences $i_{1}, \cdots, i_{p}$ of indices chosen from the set $i=1, \cdots, n-1$.

R. Gardner and P. Milanfar [9] provide an algorithm for reconstruction of an origin-symmetric convex body $\mathrm{K}$ from the volumes of its projections.

D. Ryabogin and A. Zvavich [10] reconstruct a convex body of revolution from the areas of its shadows by giving a precise formula for the support function.

In this paper, we consider a similar problem posed for the projection curvature radius function of convex bodies. We lead the problem to a system of differential equations of second order on the sphere and solve it applying a consistency method suggested by the author of the article. The solution of the system of differential equations is itself interesting.

Let $\mathbf{B} \subset \mathbf{R}^{3}$ be a convex body with sufficiently smooth boundary and with positive Gaussian curvature at every point of the boundary $\partial \mathbf{B}$. We need some notations.

$\mathrm{S}^{2}$ - the unit sphere in $\mathbf{R}^{3}, \mathrm{~S}_{\omega} \subset \mathrm{S}^{2}$-the great circle with pole at $\omega \in \mathrm{S}^{2}, \mathbf{B}(\omega)$-projection of $\mathbf{B}$ onto the plane containing the origin in $\mathbf{R}^{3}$ and orthogonal to $\omega, R\left(\omega^{\perp}, \varphi\right)$-curvature radius of $\partial \mathbf{B}(\omega)$ at the point with outer normal direction $\varphi \in \mathrm{S}_{\omega}$ and call projection curvature radius of $\mathbf{B}$.

Let $F$ be a positive continuously differentiable function defined on the space of "flags" $\mathcal{F}=\left\{(\omega, \varphi): \omega \in \mathrm{S}^{2}, \varphi \in \mathrm{S}_{\omega}\right\}$. In this article, we consider:

Problem 1. What are necessary and sufficient conditions for $F$ to be the projection curvature radius function $R\left(\omega^{\perp}, \varphi\right)$ for a convex body?

Problem 2. Reconstruction of that convex body by giving a precise formula for the support function.

Note that one can lead the problem of reconstruction of a convex body by projection curvatures using representation of the support function in terms of mean curvature radius function (see [7]). The approach of the present article is useful for practical point of view, because one can calculate curvatures of projections from the shadows of a convex body. Let's note that it is impossible to calculate mean radius of curvature from the limited number of shadows of a convex body. Also let's note that this is a different approach for such problems, because in the present article we lead the problem to a differential equation of spatial type on the sphere and solve it using a new method (so called consistency method).

The most useful analytic description of compact convex sets is by the support function (see [11]). The support function of $\mathbf{B}$ is defined as

$$
H(x)=\sup _{y \in \mathbf{B}}\langle y, x\rangle, x \in \mathbf{R}^{3} .
$$

Here $\langle\cdot, \cdot\rangle$ denotes the Euclidean scalar product in $\mathbf{R}^{3}$. The support function of $\mathbf{B}$ is positively homogeneous and convex. Below, we consider the support function $H$ of a convex body as a function on $\mathrm{S}^{2}$ (because of the positive homogeneity of $H$ the values on $\mathrm{S}^{2}$ determine $H$ completely).

$C^{k}\left(S^{2}\right)$ denotes the space of $k$ times continuously differentiable functions defined on $\mathrm{S}^{2}$. A convex body $\mathbf{B}$ is $k$-smooth if its support function $H \in C^{k}\left(\mathrm{~S}^{2}\right)$.

Given a function $H$ defined on $\mathrm{S}^{2}$, by $H_{\omega}(\varphi), \varphi \in \mathrm{S}_{\omega}$ we denote the restriction of $H$ onto the circle $\mathrm{S}_{\omega}$ for $\omega \in \mathrm{S}^{2}$, and call the restriction function of $H$.

Below, we show (Theorem 1) that Problem 1. is equivalent to the problem of existence of a function $H$ defined on $\mathrm{S}^{2}$ such that $H_{\omega}(\cdot)$ satisfies the differential equation

$$
H_{\omega}(\varphi)+\left[H_{\omega}(\varphi)\right]_{\varphi \varphi}^{\prime \prime}=F(\omega, \varphi) \text {, for } \varphi \in \mathrm{S}_{\omega}
$$

for every $\omega \in \mathrm{S}^{2}$.

Definition 1. If for a given $F$ there exists $H$ defined on $S^{2}$ that satisfies Equation (1), then $H$ is called a solution of Equation (1).

In Equation (1), $H_{\omega}(\varphi)$ is a function defined on the space of an ordered pair orthogonal unit vectors, say $e_{1}, e_{2}$, (in integral geometry such a pair is a flag and the concept of a flag was first systematically employed by 
R.V. Ambartzumian in [12]).

There are two equivalent representations of an ordered pair orthogonal unit vectors $e_{1}, e_{2}$, dual each other:

$$
(\omega, \varphi) \text { and }(\Omega, \Phi) \text {, }
$$

where $\omega \in \mathrm{S}^{2}$ is the spatial direction of the first vector $e_{1}$, and $\varphi$ is the planar direction in $\mathrm{S}_{\omega}$ coincides with the direction of $e_{2}$, while $\Omega \in \mathrm{S}^{2}$ is the spatial direction of the second vector $e_{2}$, and $\Phi$ is the planar direction in $\mathrm{S}_{\Omega}$ coincides with the direction of $e_{1}$. The second representation we will write by capital letters.

Given a flag function $g(\omega, \varphi)$, we denote by $g^{*}$ the image of $g$ defined by

$$
g^{*}(\Omega, \Phi)=g(\omega, \varphi)
$$

where $(\omega, \varphi)^{*}=(\Omega, \Phi)$ (dual each other).

Let $G$ be a function defined on $\mathcal{F}$. For every $\omega \in \mathrm{S}^{2}$, Equation (1) reduces to a differential equation on the circle $\mathrm{S}_{\omega}$.

Definition 2. If $G(\omega, \cdot)$ is a solution of that equation for every $\omega \in \mathrm{S}^{2}$, then $G$ is called a flag solution of Equation (1).

Definition 3. If a flag solution $G(\omega, \varphi)$ satisfies

$$
G^{*}(\Omega, \Phi)=G^{*}(\Omega)
$$

(no dependence on the variable $\Phi$ ), then $G$ is called a consistent flag solution.

There is an important principle: each consistent flag solution $G$ of Equation (1) produces a solution of Equation (1) via the map

$$
G(\omega, \varphi) \rightarrow G^{*}(\Omega, \Phi)=G^{*}(\Omega)=H(\Omega),
$$

and vice versa: the restriction functions of any solution of Equation (1) onto the great circles is a consistent flag solution.

Hence, the problem of finding a solution reduces to finding a consistent flag solution.

To solve the latter problem, the present paper applies the consistency method first used in [13]-[15] in an integral equations context.

We denote: $e[\Omega, \Phi]$ 一 the plane containing the origin of $\mathbf{R}^{3}$, direction $\Omega \in \mathrm{S}^{2}, \Phi$ determines rotation of the plane around $\Omega, \mathbf{B}[\Omega, \Phi]$-projection of $\mathbf{B} \in \mathcal{B}$ onto the plane $e[\Omega, \Phi], R^{*}(\Omega, \Phi)$-curvature radius of $\partial \mathbf{B}[\Omega, \Phi]$ at the point with outer normal direction $\Omega \in \mathrm{S}^{2}$. It is easy to see that

$$
R^{*}(\Omega, \Phi)=R\left(\omega^{\perp}, \varphi\right)
$$

where $(\Omega, \Phi)$ is dual to $(\omega, \varphi)$.

Note that in the Problem 1. uniqueness (up to a translation) follows from the classical uniqueness result on Christoffel problem, since

$$
R_{1}(\Omega)+R_{2}(\Omega)=\frac{1}{\pi} \int_{0}^{2 \pi} R^{*}(\Omega, \Phi) \mathrm{d} \Phi
$$

Equation (1) has the following geometrical interpretation.

It is known (see [11]) that 2 times continuously differentiable homogeneous function $H$ defined on $\mathbf{R}^{3}$, is convex if and only if

$$
H_{\omega}(\varphi)+\left[H_{\omega}(\varphi)\right]_{\varphi \varphi}^{\prime \prime} \geq 0 \text { for every } \omega \in \mathrm{S}^{2} \text { and } \varphi \in S_{\omega},
$$

where $H_{\omega}(\cdot)$ is the restriction of $H$ onto $\mathrm{S}_{\omega}$.

So in case $F>0$, it follows from (7), that if $H$ is a solution of Equation (1) then its homogeneous extension is convex.

It is known from convexity theory that if a homogeneous function $H$ is convex then there is a unique convex body $\mathbf{B} \subset \mathbf{R}^{3}$ with support function $H$ and $F(\omega, \varphi)$ is the projection curvature radius function of $\mathbf{B}$ (see [11]).

The support function of each parallel shifts (translation) of that body $\mathbf{B}$ will again be a solution of Equation (1). By uniqueness, every two solutions of Equation (1) differ by a summand $\langle a, \cdot\rangle$ defined on $S^{2}$, where 
$a \in \mathbf{R}^{3}$. Thus we have the following theorem.

Theorem 1 Let $F$ be a positive function defined on $\mathcal{F}$. If Equation (1) has a solution $H$ then there exists a convex body $\mathbf{B}$ with projection curvature radius function $F$, whose support function is $H$. Every solution of Equation (1) has the form $H(\cdot)+\langle a, \cdot\rangle$, where $a \in \mathbf{R}^{3}$, being the support function of the convex body $\mathbf{B}+a$.

The converse statement is also true. The support function $H$ of a 2-smooth convex body $\mathbf{B}$ satisfies Equation (1) for $F=R$, where $R$ is the projection curvature radius function of $\mathbf{B}$ (see [16]).

The purpose of the present paper is to find a necessary and sufficient condition that ensures a positive answer to both Problems 1,2 and suggest an algorithm of construction of the body $\mathbf{B}$ by finding a representation of the support function in terms of projection curvature radius function. This happens to be a solution of Equation (1).

Throughout the paper (in particular, in Theorem 2 that follows) we use usual spherical coordinates $v, \tau$ for points $\mathrm{S}^{2}$ based on a choice of a North Pole and a reference point $\tau=0$ on the equator. The point with coordinates $v, \tau$ we will denote by $(v, \tau)$, the points $(0, \tau)$ lie on the equator. On $\mathrm{S}_{\omega}$ we choose anticlockwise direction as positive. On the plane $\omega^{\perp}$ containing $\mathrm{S}_{\omega}$ we consider the Cartesian $\mathrm{x}$ and y-axes where the direction of the y-axis $\vec{y}$ is taken to be the projection of the North Pole onto $\omega^{\perp}$. The direction of the x-axis $x$ we take as the reference direction on $\mathrm{S}_{\omega}$ and call it the East direction. Now we describe the main result.

Theorem 2 Let $\mathbf{B}$ be a 3-smooth convex body with positive Gaussian curvature at every point of $\partial \mathbf{B}$ and $R$ is the projection curvature radius function of $\mathbf{B}$. Then for $\Omega \in \mathrm{S}^{2}$ chosen as the North pole

$$
\begin{aligned}
H(\Omega)= & \frac{1}{4 \pi} \int_{0}^{2 \pi}\left[\int_{0}^{\frac{\pi}{2}} R\left((0, \tau)^{\perp}, \varphi\right) \cos \varphi \mathrm{d} \varphi\right] \mathrm{d} \tau \\
& +\frac{1}{8 \pi^{2}} \int_{0}^{2 \pi}\left[\int_{-\frac{\pi}{2}}^{\frac{\pi}{2}} R\left((0, \tau)^{\perp}, \varphi\right)\left((\pi+2 \varphi) \cos \varphi-2 \sin ^{3} \varphi\right) \mathrm{d} \varphi\right] \mathrm{d} \tau \\
& -\frac{1}{2 \pi^{2}} \int_{0}^{\frac{\pi}{2}} \frac{\sin v}{\cos ^{2} v} \mathrm{~d} v \int_{0}^{2 \pi} \mathrm{d} \tau \int_{0}^{2 \pi} R\left((v, \tau)^{\perp}, \varphi\right) \sin ^{3} \varphi \mathrm{d} \varphi
\end{aligned}
$$

is a solution of Equation (1) for $F=R$. On $\mathrm{S}_{\omega}$ we measure $\varphi$ from the East direction.

Remark, that the order of integration in the last integral of (8) cannot be changed.

Obviously Theorem 2 suggests a practical algorithm of reconstruction of convex body from projection curvature radius function $R$ by calculation of support function $H$.

We turn to Problem 1. Let $R$ be the projection curvature radius function of a convex body $\mathbf{B}$. Then $F \equiv R$ necessarily satisfies the following conditions:

a) For every $\omega \in \mathrm{S}^{2}$ and any reference point on $\mathrm{S}_{\omega}$

$$
\int_{0}^{2 \pi} F(\omega, \varphi) \sin \varphi \mathrm{d} \varphi=\int_{0}^{2 \pi} F(\omega, \varphi) \cos \varphi \mathrm{d} \varphi=0 .
$$

This follows from Equation (1), see also [16].

b) For every direction $\Omega \in \mathrm{S}^{2}$ chosen as the North pole

$$
\int_{0}^{2 \pi}\left[F^{*}((v, \tau), y)\right]_{v=0}^{\prime} \mathrm{d} \tau=0,
$$

where the function $F^{*}$ is the image of $F$ (see (3)) and $y$ is the direction of the y-axis on $(v, \tau)^{\perp}$ (Theorem 5).

Let $F$ be a positive 2 times differentiable function defined on $\mathcal{F}$. Using (8), we construct a function $\bar{F}$ defined on $S^{2}$ :

$$
\begin{aligned}
\bar{F}(\Omega)= & \frac{1}{4 \pi} \int_{0}^{2 \pi}\left[\int_{0}^{\frac{\pi}{2}} F((0, \tau), \varphi) \cos \varphi \mathrm{d} \varphi\right] \mathrm{d} \tau \\
& +\frac{1}{8 \pi^{2}} \int_{0}^{2 \pi}\left[\int_{-\frac{\pi}{2}}^{\frac{\pi}{2}} F((0, \tau), \varphi)\left((\pi+2 \varphi) \cos \varphi-2 \sin ^{3} \varphi\right) \mathrm{d} \varphi\right] \mathrm{d} \tau \\
& -\frac{1}{2 \pi^{2}} \int_{0}^{\frac{\pi}{2}} \frac{\sin v}{\cos ^{2} v} \mathrm{~d} v \int_{0}^{2 \pi} \mathrm{d} \tau \int_{0}^{2 \pi} F((v, \tau), \varphi) \sin ^{3} \varphi \mathrm{d} \varphi
\end{aligned}
$$

Note that the last integral converges if the condition (10) is satisfied. 
Theorem 3 A positive 2 times differentiable function $F$ defined on $\mathcal{F}$ represents the projection curvature radius function of some convex body $\mathbf{B}$ if and only if $F$ satisfies the conditions (9), (10) and the extension (to $\mathbf{R}^{3}$ ) of the function $F$ defined by (11) is convex.

\section{The Consistency Condition}

We fix $\omega \in \mathrm{S}^{2}$ and try to solve Equation (1) as a differential equation of second order on the circle $\mathrm{S}_{\omega}$. We start with two results from [16].

a) For any smooth convex domain $D$ in the plane

$$
h(\varphi)=\int_{0}^{\varphi} R(\psi) \sin (\varphi-\psi) \mathrm{d} \psi,
$$

where $h(\varphi)$ is the support function of $D$ with respect to a point $s \in \partial D$. In (12) we measure $\varphi$ from the normal direction at s, $R(\psi)$ is the curvature radius of $\partial D$ at the point with normal direction $\psi$.

b) (12) is a solution of the following differential equation

$$
R(\varphi)=h(\varphi)+h^{\prime \prime}(\varphi) .
$$

One can easy verify that (also it follows from (13) and (12))

$$
G(\omega, \varphi)=\int_{0}^{\varphi} F(\omega, \psi) \sin (\varphi-\psi) \mathrm{d} \psi,
$$

is a flag solution of Equation (1).

Theorem 4 Every flag solution of Equation (1) has the form

$$
g(\omega, \varphi)=\int_{0}^{\varphi} F(\omega, \psi) \sin (\varphi-\psi) \mathrm{d} \psi+C(\omega) \cos \varphi+S(\omega) \sin \varphi
$$

where $C_{n}$ and $S_{n}$ are some real coefficients.

Proof of Theorem 4. Every continuous flag solution of Equation (1) is a sum of $G+g_{0}$, where $g_{0}$ is a flag solution of the corresponding homogeneous equation:

$$
H_{\omega}(\varphi)+\left[H_{\omega}(\varphi)\right]_{\varphi \varphi}^{\prime \prime}=0, \varphi \in \mathrm{S}_{\omega},
$$

for every $\omega \in \mathrm{S}^{2}$. We look for the general flag solution of Equation (16) in the form of a Fourier series

$$
g_{0}(\omega, \varphi)=\sum_{n=0,1,2, \cdots}\left[C_{n}(\omega) \cos n \varphi+S_{n}(\omega) \sin n \varphi\right] .
$$

After substitution of (17) into (16) we obtain that $g_{0}(\omega, \varphi)$ satisfies (16) if and only if

$$
g_{0}(\omega, \varphi)=C_{1}(\omega) \cos \varphi+S_{1}(\omega) \sin \varphi .
$$

Now we try to find functions $C$ and $S$ in (15) from the condition that $g$ satisfies (4). We write $g(\omega, \varphi)$ in dual coordinates i.e. $g(\omega, \varphi)=g^{*}(\Omega, \Phi)$ and require that $g^{*}(\Omega, \Phi)$ should not depend on $\Phi$ for every $\Omega \in \mathrm{S}^{2}$, i.e. for every $\Omega \in \mathrm{S}^{2}$

$$
\left(g^{*}(\Omega, \Phi)\right)_{\Phi}^{\prime}=(G(\omega, \varphi)+C(\omega) \cos \varphi+S(\omega) \sin \varphi)_{\Phi}^{\prime}=0,
$$

where $G(\omega, \varphi)$ was defined in (14).

Here and below $(\cdot)_{\Phi}^{\prime}$ denotes the derivative corresponding to right screw rotation around $\Omega$. Differentiation with use of expressions (see [14])

$$
\tau_{\Phi}^{\prime}=\frac{\sin \varphi}{\cos v}, \varphi_{\Phi}^{\prime}=-\tan v \sin \varphi, v_{\Phi}^{\prime}=-\cos \varphi,
$$

after a natural grouping of the summands in (18), yields the Fourier series of $-(G(\omega, \varphi))_{\Phi}^{\prime}$. By uniqueness of the Fourier coefficients 


$$
\begin{gathered}
(C(\omega))_{v}^{\prime}+\frac{(S(\omega))_{\tau}^{\prime}}{\cos v}+\tan v C(\omega)=\frac{1}{\pi} \int_{0}^{2 \pi} A(\omega, \varphi) \cos 2 \varphi \mathrm{d} \varphi \\
(C(\omega))_{v}^{\prime}-\frac{(S(\omega))_{\tau}^{\prime}}{\cos v}-\tan v C(\omega)=\frac{1}{2 \pi} \int_{0}^{2 \pi} A(\omega, \varphi) \mathrm{d} \varphi \\
(S(\omega))_{v}^{\prime}-\frac{(C(\omega))_{\tau}^{\prime}}{\cos v}+\tan v S(\omega)=\frac{1}{\pi} \int_{0}^{2 \pi} A(\omega, \varphi) \sin 2 \varphi \mathrm{d} \varphi,
\end{gathered}
$$

where

$$
A(\omega, \varphi)=\int_{0}^{\varphi}\left[F(\omega, \psi)_{\Phi}^{\prime} \sin (\varphi-\psi)+F(\omega, \psi) \cos (\varphi-\psi) \varphi_{\Phi}^{\prime}\right] \mathrm{d} \psi
$$

\section{Averaging}

Let $H$ be a solution of Equation (1), i.e. restriction of $H$ onto the great circles is a consistent flag solution of Equation (1). By Theorem 1 there exists a convex body $\mathbf{B} \in \mathcal{B}$ with projection curvature radius function $R=F$, whose support function is $H$.

To calculate $H(\Omega)$ for a $\Omega \in \mathrm{S}^{2}$ we take $\Omega$ for the North Pole of $\mathrm{S}^{2}$. Returning to the Formula (15) for every $\omega=(0, \tau) \in \mathrm{S}_{\Omega}$ we have

$$
H(\Omega)=\int_{0}^{\frac{\pi}{2}} R\left(\omega^{\perp}, \psi\right) \sin \left(\frac{\pi}{2}-\psi\right) \mathrm{d} \psi+S(\omega),
$$

We integrate both sides of (22) with respect to uniform angular measure $\mathrm{d} \tau$ over $[0,2 \pi)$ to get

$$
2 \pi H(\Omega)=\int_{0}^{2 \pi} \int_{0}^{\frac{\pi}{2}} R\left((0, \tau)^{\perp}, \psi\right) \cos \psi \mathrm{d} \psi \mathrm{d} \tau+\int_{0}^{2 \pi} S((0, \tau)) \mathrm{d} \tau .
$$

Now the problem is to calculate

$$
\int_{0}^{2 \pi} S((0, \tau)) \mathrm{d} \tau=\bar{S}(0)
$$

We are going to integrate both sides of (20) and (21) with respect to $\mathrm{d} \tau$ over $[0,2 \pi)$. For $\omega=(v, \tau)$, where $v \in\left[0, \frac{\pi}{2}\right)$ and $\tau \in(0,2 \pi)$ we denote

$$
\begin{gathered}
\bar{S}(v)=\int_{0}^{2 \pi} S((v, \tau)) \mathrm{d} \tau, \\
\pi A(v)=\int_{0}^{2 \pi} \mathrm{d} \tau \int_{0}^{2 \pi}\left[\int_{0}^{\varphi}\left[R\left(\omega^{\perp}, \psi\right)_{\Phi}^{\prime} \sin (\varphi-\psi)+R\left(\omega^{\perp}, \psi\right) \cos (\varphi-\psi) \varphi_{\Phi}^{\prime}\right] \mathrm{d} \psi\right] \sin 2 \varphi \mathrm{d} \varphi .
\end{gathered}
$$

Integrating both sides of (20) and (21) and taking into account that

$$
\int_{0}^{2 \pi}(C(v, \tau))_{\tau}^{\prime} \mathrm{d} \tau=0
$$

for $v \in[0, \pi / 2)$ we get

$$
\overline{S^{\prime}}(v)+\tan v \bar{S}(v)=A(v),
$$

i.e. a differential equation for the unknown coefficient $\bar{S}(v)$.

We have to find $\bar{S}(0)$ given by (24). It follows from (27) that

$$
\left(\frac{\bar{S}(v)}{\cos v}\right)=\frac{A(v)}{\cos v} .
$$


Integrating both sides of (5.1) with respect to $\mathrm{d} v$ over $[0, \pi / 2)$ we obtain

$$
\bar{S}(0)=\left.\frac{\bar{S}(v)}{\cos v}\right|_{\frac{\pi}{2}}-\int_{0}^{\frac{\pi}{2}} \frac{A(v)}{\cos v} \mathrm{~d} v .
$$

Now, we are going to calculate $\left.\frac{\bar{S}(v)}{\cos v}\right|_{\frac{\pi}{2}}$.

It follows from (15) that

$$
\begin{aligned}
\pi \bar{S}(v) & \left.=\int_{0}^{2 \pi} \int_{0}^{2 \pi}\left[H_{\omega}(\varphi)-\int_{0}^{\varphi} R\left(\omega^{\perp}, \psi\right)\right) \sin (\varphi-\psi) \mathrm{d} \psi\right] \sin \varphi \mathrm{d} \varphi \mathrm{d} \tau \\
& =\int_{0}^{2 \pi} \int_{0}^{2 \pi} H_{\omega}(\varphi) \sin \varphi \mathrm{d} \varphi \mathrm{d} \tau-\frac{1}{2} \int_{0}^{2 \pi} \int_{0}^{2 \pi} R\left(\omega^{\perp}, \psi\right)((2 \pi-\psi) \cos \psi+\sin \psi) \mathrm{d} \psi \mathrm{d} \tau .
\end{aligned}
$$

Let $\varphi \in \mathrm{S}_{\omega}$ be the direction that corresponds to $\varphi \in[0,2 \pi)$, for $\omega=(v, \tau)$. As a point of $\mathrm{S}^{2}$, let $\varphi$ have spherical coordinates $u, t$ with respect to $\Omega$. By the sinus theorem of spherical geometry

$$
\cos v \sin \varphi=\sin u \text {. }
$$

From (31), we get

$$
(u)_{v=\frac{\pi}{2}}^{\prime}=-\sin \varphi .
$$

Fixing $\tau$ and using (32) we write a Taylor formula at a neighborhood of the point $v=\pi / 2$ :

$$
H_{(v, \tau)}(\varphi)=H((0, \varphi+\tau))+H_{v}^{\prime}((0, \varphi+\tau)) \sin \varphi\left(\frac{\pi}{2}-v\right)+o\left(\frac{\pi}{2}-v\right) .
$$

Similarly, for $\psi \in[0,2 \pi)$ we get

$$
\begin{aligned}
R\left((v, \tau)^{\perp}, \psi\right) & =R\left(\left(\frac{\pi}{2}, \tau\right)^{\perp}, \psi+\tau\right) \\
& +R_{\nu}^{\prime}\left(\left(\frac{\pi}{2}, \tau\right)^{\perp}, \psi+\tau\right) \sin \psi\left(\frac{\pi}{2}-v\right)+o\left(\frac{\pi}{2}-v\right) .
\end{aligned}
$$

Substituting (33) and (34) into (30) and taking into account the easily establish equalities

$$
\int_{0}^{2 \pi} \int_{0}^{2 \pi} H((0, \varphi+\tau)) \sin \varphi \mathrm{d} \varphi \mathrm{d} \tau=0
$$

and

$$
\int_{0}^{2 \pi} \int_{0}^{2 \pi} R\left(\left(\frac{\pi}{2}, \tau\right)^{\perp}, \psi+\tau\right)((2 \pi-\psi) \cos \psi+\sin \psi) \mathrm{d} \psi \mathrm{d} \tau=0
$$

we obtain

$$
\begin{aligned}
\lim _{v \rightarrow \frac{\pi}{2}} \frac{\bar{S}(v)}{\cos v}= & \frac{1}{\pi} \int_{0}^{2 \pi} \int_{0}^{2 \pi} H_{v}^{\prime}((0, \varphi+\tau)) \sin ^{2} \varphi \mathrm{d} \varphi \mathrm{d} \tau \\
& \left.-\frac{1}{2 \pi} \int_{0}^{2 \pi} \int_{0}^{2 \pi} R_{v}^{\prime}\left(\left(\frac{\pi}{2}, \tau\right)\right)^{\perp}, \psi+\tau\right) \sin \psi((2 \pi-\psi) \cos \psi+\sin \psi) \mathrm{d} \psi \mathrm{d} \tau \\
= & \int_{0}^{2 \pi} H_{v}^{\prime}((0, \tau)) \mathrm{d} \tau-\frac{3}{4} \int_{0}^{2 \pi}\left[R^{*}((v, \tau), y)\right]_{v=0}^{\prime} \mathrm{d} \tau .
\end{aligned}
$$

Theorem 5 For every 3-smooth convex body $\mathbf{B} \in \mathcal{B}$ and any direction $\Omega \in \mathrm{S}^{2}$, we have 


$$
\int_{0}^{2 \pi}\left[R^{*}((v, \tau), y)\right]_{v=0}^{\prime} \mathrm{d} \tau=0,
$$

where $y$ is the direction of the $y$-axis on $(v, \tau)^{\perp}$.

Proof of Theorem 5. Using spherical geometry, one can prove that (see also (1))

$$
\begin{aligned}
{\left[R^{*}((v, \tau), y)\right]_{v=0}^{\prime} } & =\left[H((v, \tau))+H_{\varphi \varphi}^{\prime \prime}((v, \tau))\right]_{v=0} \\
& =\left[H((v, \tau))+H_{\tau \tau}^{\prime \prime} \frac{1}{\cos ^{2} v}-H_{v}^{\prime} \tan v\right]_{v=0} \\
& =\left[H_{\tau \tau}^{\prime \prime}\right]_{v=0}^{\prime},
\end{aligned}
$$

where $H$ is the support function of $\mathbf{B}$. Integrating (38), we get

$$
\int_{0}^{2 \pi}\left[R^{*}((v, \tau), y)\right]_{v=0}^{\prime} \mathrm{d} \tau=\int_{0}^{2 \pi}\left[H_{\tau \tau}^{\prime \prime}\right]_{v=0}^{\prime} \mathrm{d} \tau=0
$$

\section{A Representation for Support Functions of Convex Bodies}

Let $\mathbf{B} \in \mathcal{B}$ be a convex body and $Q \in \mathbf{R}^{3}$. By $H_{Q}$ we denote the support function of $\mathbf{B}$ with respect to $Q$.

Theorem 6 Given a 2-smooth convex body $\mathbf{B} \in \mathcal{B}$, there exists a point $O^{*} \in \mathbf{R}^{3}$ such that for every $\Omega \in \mathrm{S}^{2}$ chosen as the North pole

$$
\int_{0}^{2 \pi}\left[H_{O^{*}}((v, \tau))\right]_{v=0} \mathrm{~d} \tau=0 .
$$

Proof of Theorem 6. For a given $\mathbf{B}$ and a point $Q \in \mathbf{R}^{3}$, by $K_{Q}$ we denote the following function defined on $\mathrm{S}^{2}$

$$
K_{Q}(\Omega)=\int_{0}^{2 \pi}\left[H_{Q}((v, \tau))\right]_{v=0} \mathrm{~d} \tau .
$$

Clearly, $K_{Q}$ is a continuous odd function with maximum $\bar{K}(Q)$ :

$$
\bar{K}(Q)=\max _{\Omega \in S^{2}} K_{Q}(\Omega) .
$$

It is easy to see that $\bar{K}(Q) \rightarrow \infty$ for $|Q| \rightarrow \infty$. Since $\bar{K}(Q)$ is continuous, so there is a point $O^{*}$ for which

$$
\bar{K}\left(O^{*}\right)=\min \bar{K}(Q) .
$$

Let $\Omega^{*}$ be a direction of maximum now assumed to be unique, i.e.

$$
\bar{K}\left(O^{*}\right)=\max _{\Omega \in \mathrm{S}^{2}} K_{O^{*}}(\Omega)=K_{O^{*}}\left(\Omega^{*}\right) .
$$

If $\bar{K}\left(O^{*}\right)=0$ the theorem is proved. For the case $\bar{K}\left(O^{*}\right)=a>0$ let $O^{* *}$ be the point for which $\boldsymbol{O}^{*} \boldsymbol{O}^{* *}=\varepsilon \Omega^{*}$. It is easy to demonstrate that $H_{O^{* *}}(\Omega)=H_{O^{*}}(\Omega)-\varepsilon\left(\Omega, \Omega^{*}\right)$, hence for a small $\varepsilon>0$ we find that $\bar{K}\left(O^{* *}\right)=a-2 \pi \varepsilon$, contrary to the definition of $O^{*}$. So $\bar{K}\left(O^{*}\right)=0$. For the case where there are two or more directions of maximum one can apply a similar argument. $H$.

Now we take the point $O^{*}$ of the convex body $\mathbf{B}$ for the origin of $\mathbf{R}^{3}$. Below $H_{O^{*}}$, we will simply denote by

By Theorem 6 and Theorem 5, we have the boundary condition (see (36))

$$
\left.\frac{\bar{S}(v)}{\cos v}\right|_{\frac{\pi}{2}}=0 .
$$

Substituting (29) into (23) we get 


$$
\begin{aligned}
2 \pi H(\Omega)= & \int_{0}^{2 \pi} \int_{0}^{\frac{\pi}{2}} R\left((0, \tau)^{\perp}, \psi\right) \cos \psi \mathrm{d} \psi \mathrm{d} \tau-\int_{0}^{\frac{\pi}{2}} \frac{A(v)}{\cos v} \mathrm{~d} v \\
= & \int_{0}^{2 \pi} \int_{0}^{\frac{\pi}{2}} R\left((0, \tau)^{\perp}, \psi\right) \cos \psi \mathrm{d} \psi \mathrm{d} \tau-\frac{1}{\pi} \int_{0}^{\frac{\pi}{2}} \frac{\mathrm{d} v}{\cos v} \\
& \times \int_{0}^{2 \pi} \int_{0}^{2 \pi}\left[\int_{0}^{\varphi}\left[R\left(\omega^{\perp}, \psi\right)_{\Phi}^{\prime} \sin (\varphi-\psi)+R\left(\omega^{\perp}, \psi\right) \cos (\varphi-\psi) \varphi_{\Phi}^{\prime}\right] \mathrm{d} \psi\right] \sin 2 \varphi \mathrm{d} \varphi \mathrm{d} \tau .
\end{aligned}
$$

Using expressions (19) and integrating by $\mathrm{d} \varphi$ yields

$$
\begin{aligned}
2 \pi H(\Omega)= & \int_{0}^{2 \pi} \int_{0}^{\frac{\pi}{2}} R\left((0, \tau)^{\perp}, \psi\right) \cos \psi \mathrm{d} \psi \mathrm{d} \tau \\
& +\frac{1}{\pi} \int_{0}^{\frac{\pi}{2}} \frac{\mathrm{d} v}{\cos v} \int_{0}^{2 \pi} \mathrm{d} \tau \int_{0}^{2 \pi}\left[R\left(\omega^{\perp}, \psi\right)_{v}^{\prime} I+R\left(\omega^{\perp}, \psi\right) \tan v I I\right] \mathrm{d} \psi,
\end{aligned}
$$

where

$$
I I=\int_{\psi}^{2 \pi} \sin 2 \varphi \cos (\varphi-\psi) \sin \varphi \mathrm{d} \varphi=\left[\frac{(2 \pi-\psi) \cos \psi}{4}+\frac{\sin \psi\left(1+\sin ^{2} \psi\right)}{4}-\sin ^{3} \psi\right]
$$

and

$$
I=\int_{\psi}^{2 \pi} \sin 2 \varphi \sin (\varphi-\psi) \cos \varphi \mathrm{d} \varphi=\left[\frac{(2 \pi-\psi) \cos \psi}{4}+\frac{\sin \psi\left(1+\sin ^{2} \psi\right)}{4}\right]
$$

Integrating by parts (42) we get

$$
\begin{aligned}
2 \pi H(\Omega)= & \int_{0}^{2 \pi} \int_{0}^{\frac{\pi}{2}} R\left((0, \tau)^{\perp}, \psi\right) \cos \psi \mathrm{d} \psi \mathrm{d} \tau-\frac{1}{\pi} \int_{0}^{\frac{\pi}{2}} \mathrm{~d} v \int_{0}^{2 \pi} \mathrm{d} \tau \int_{0}^{2 \pi} R\left(\omega^{\perp}, \psi\right) \frac{\sin v \sin ^{3} \psi}{\cos ^{2} v} \mathrm{~d} \psi \\
& -\frac{1}{\pi} \int_{0}^{\frac{\pi}{2}} \mathrm{~d} \tau \int_{0}^{2 \pi} R\left((0, \tau)^{\perp}, \psi\right) I \mathrm{~d} \psi+\lim _{a \rightarrow \frac{\pi}{2}} \frac{1}{\pi \cos a} \int_{0}^{\frac{\pi}{2}} \mathrm{~d} \tau \int_{0}^{2 \pi} R\left((a, \tau)^{\perp}, \psi\right) I \mathrm{~d} \psi
\end{aligned}
$$

Using (34), Theorem 5 and taking into account that

$$
\int_{0}^{2 \pi} I \mathrm{~d} \psi=0
$$

we get

$$
\begin{aligned}
2 \pi H(\Omega)= & \int_{0}^{2 \pi} \int_{0}^{\frac{\pi}{2}} R\left((0, \tau)^{\perp}, \psi\right) \cos \psi \mathrm{d} \psi \mathrm{d} \tau-\frac{1}{\pi} \int_{0}^{\frac{\pi}{2}} \mathrm{~d} v \int_{0}^{2 \pi} \mathrm{d} \tau \int_{0}^{2 \pi} R\left(\omega^{\perp}, \psi\right) \frac{\sin v \sin ^{3} \psi}{\cos ^{2} \nu} \mathrm{d} \psi \\
& -\frac{1}{\pi} \int_{0}^{\frac{\pi}{2}} \mathrm{~d} \tau \int_{0}^{2 \pi} R\left((0, \tau)^{\perp}, \psi\right) I \mathrm{~d} \psi .
\end{aligned}
$$

From (44), using (9) we obtain (8). Theorem 2 is proved.

\section{Proof of Theorem 3}

Necessity: if $F$ is the projection curvature radius function of a convex body $\mathbf{B} \in \mathcal{B}$, then it satisfies (9) (see [16]), the condition (10) (Theorem 5) and $F$ defined by (11) is convex since it is the support function of $\mathbf{B}$ (Theorem 2).

Sufficiency: let $F$ be a positive 2 times differentiable function defined on $\mathcal{F}$ satisfies the conditions (9), (10). We construct the function $F$ on $S^{2}$ defined by (11). There exists a convex body $\mathbf{B}$ with support function $F$ since its extension is a convex function. Also Theorem 2 implies that $F$ is the projection curvature radius of $\mathbf{B}$.

\section{Funding}

This work was partially supported by State Committee Science MES RA, in frame of the research project SCS 
13-1A244.

\section{References}

[1] Minkowski, H. (1911) Theorie der konvexen Korper, insbesondere Begrundung ihresb Oberflachenbergriffs. Ges. Abh., 2, Leipzig, Teubner, 131-229.

[2] Blaschke, W. (1923) Vorlesungen uber Differentialgeometrie. II. Affine Differentialgeometrie, Springer-Verlag, Berlin.

[3] Pogorelov, A.V. (1969) Exterior Geometry of Convex Surfaces [in Russian]. Nauka, Moscow.

[4] Alexandrov, A.D. (1956) Uniqueness Theorems for Surfaces in the Large [in Russian]. Vesti Leningrad State University, 19, 25-40.

[5] Bakelman, I.Ya., Verner, A.L. and Kantor, B.E. (1973) Differential Geometry in the Large [in Russian]. Nauka, Moskow.

[6] Firey, W.J. (1970) Intermediate Christoffel-Minkowski Problems for Figures of Revolution. Israel Journal of Mathematics, 8, 384-390. http://dx.doi.org/10.1007/BF02798684

[7] Berg, C. (1969) Corps convexes et potentiels spheriques. Matematisk-fysiske Meddelelser Udgivet af. Det Kongelige Danske Videnskabernes Selska, 37, 64.

[8] Wiel, W. and Schneider, R. (1983) Zonoids and Related Topics. In: Gruber, P. and Wills, J., Eds., Convexity and Its Applications, Birkhauser, Basel, 296-317.

[9] Gardner R.J. and Milanfar, P. (2003) Reconstruction of Convex Bodies from Brightness Functions. Discrete \& Computational Geometry, 29, 279-303. http://dx.doi.org/10.1007/s00454-002-0759-2

[10] Ryabogin, D. and Zvavich, A. (2004) Reconstruction of Convex Bodies of Revolution from the Areas of Their Shadows. Archiv der Mathematik, 5, 450-460. http://dx.doi.org/10.1007/s00454-002-0759-2

[11] Leichtweiz, K. (1980) Konvexe Mengen, VEB Deutscher Verlag der Wissenschaften, Berlin. http://dx.doi.org/10.1007/978-3-642-95335-4

[12] Ambartzumian, R.V. (1990) Factorization Calculus and Geometrical Probability. Cambridge University Press, Cambridge. http://dx.doi.org/10.1017/CBO9781139086561

[13] Aramyan, R.H. (2001) An Approach to Generalized Funk Equations I [in Russian]. Izvestiya Akademii Nauk Armenii. Matematika [English Translation: Journal of Contemporary Mathematical Analysis (Armenian Academy of Sciences)], 36, 47-58.

[14] Aramyan, R.H. (2010) Generalized Radon Transform on the Sphere. Analysis International Mathematical Journal of Analysis and Its Applications, 30, 271-284.

[15] Aramyan, R.H. (2010) Solution of an Integral Equation by Consistency Method. Lithuanian Mathematical Journal, 50, 133-139.

[16] Blaschke, W. (1956) Kreis und Kugel, (Veit, Leipzig). 2nd Edition, De Gruyter, Berlin. 